\title{
Weniger gastrointestiale Tumoren nach Hormonersatztherapie
}

\begin{abstract}
Das erhöhte Brustkrebsrisiko einer Hormonersatztherapie (HRT) hat zu einem zurückhaltenden Einsatz dieser Behandlung geführt. Daran wird auch die Nachricht, dass eine HRT das Risiko für Malignome des Gastrointestinaltrakts senkt, nichts ändern.
\end{abstract}

B islang war bekannt, dass eine HRT das Risiko für Darmkrebs senkt. Über das Risiko für Ösophagus- und Magenkarzinome gab es dagegen nur wenige Angaben. Deshalb wurde in einer FallKontroll-Studie die Häufigkeit auch dieser malignen Tumoren untersucht und die Daten in einer Metaanalyse zusammen mit bisher publizierten Studien zu dieser Fragestellung analysiert. Die Kohorte umfasste Frauen im Alter von $\geq 50$ Jahren aus einer Datenbank hausärztlicher Patienten in Großbritannien. Darunter waren 1.054 Fälle von Ösophaguskarzinomen, $750 \mathrm{mit}$ Magenkrebs und 4.708 mit kolorektalen
Karzinomen. Jeder Tumorpatientin wurden jeweils fünf nach Alter und Praxis passende Patientinnen zugeordnet und das relative Risiko (RR) bei HRT anhand prospektiv erfasster HRT-Verordnung ermittelt.

Frauen, denen mindestens einmal eine HRT verschrieben worden war, hatten ein um $32 \%$ signifikant geringeres Risiko für ein Ösophaguskarzinom als Frauen ohne HRT-Verordnung (RR 0,68; 95\%-Konfidenzintervall $[95 \%-\mathrm{KI}]$ 0,53-0,88; $\mathrm{p}=$ $0,004)$. Auch das Magenkrebs-Risiko verringerte sich bei mindestens einmaliger HRT-Verordnung um $25 \%$, allerdings war der Unterschied hier nicht signifikant (RR 0,$75 ; 95 \%$-KI 0,54-1,05; p = 0,1). Schließlich fanden die Wissenschaftler auch in dieser Kohorte ein signifikant um 19\% reduziertes Darmkrebsrisiko (RR 0,81; 95\%-KI 0,73-0,90; p < 0,001). Art und Dauer der Hormonersatztherapie spielte dabei ebensowenig eine Rolle wie die Unterscheidung zwischen aktueller und früherer Einnahme. In der Metaanalyse zeigte sich für alle drei Karzinom-Entitäten ein signifikant reduziertes Risiko bei HRT mit einem RR von 0,68 für Ösophagus- $(\mathrm{p}<0,001), 0,78$ für Magen- $(\mathrm{p}=$ $0,008)$ und 0,84 für kolorektale Karzinome $(\mathrm{p}<0,001)$. Absolut sind die Unterschiede aber überschaubar: Für Länder mit hohem Einkommen errechneten die Autoren für Frauen im Alter von 50 bis 64 Jahren ein Risiko für alle drei Karzinomentitäten über fünf Jahre von 2,9 pro 1.000 , wenn sie HRT nutzten, und 3,6 pro 1.000, wenn sie nie eine HRT verordnet bekommen hatten.

Fazit: Eine Hormonersatztherapie verringert das Risiko für Ösophagus-, Magenund Darmkarzinome signifikant. Allerdings ist dieser Effekt im Vergleich zu der Risikoerhöhung beim Mammakarzinom gering.

Friederike Klein

Green J et al. Menopausal hormone therapy and risk of gastrointestinal cancer: nested case-control study within a prospective cohort, and meta-analysis. Int J Cancer. 2012;130(10):2387-96.

\section{Stuhltest und Koloskopie anbieten}

\section{Mit welcher Strategie man am meisten Menschen für die Teilnahme am Darmkrebs-Screening gewinnen kann - darüber haben sich schon viele Ärzte den Kopf zerbrochen. Eine Studie schafft nun etwas mehr Klarheit.}

D er Test auf okkultes Blut im Stuhl ist zwar unkomplizierter und einfacher, präzisere Ergebnisse liefert bekanntlich die Koloskopie. Die ist jedoch nicht gerade beliebt. Soll man also lieber die einfachere Methode empfehlen, um damit möglichst viele Menschen zu erreichen, oder auf die präzisere Methode setzen und dabei in Kauf nehmen, dass nur wenige die Prozedur akzeptieren? Eine Lösung dafür haben nun offenbar US-Gastroenterologen gefunden: Am besten beides anbieten. Das schließen sie zumindest aus den Ergebnissen einer dreiarmigen Studie mit knapp 1.000 Teilnehmern, die zum Screening eingeladen wurden. Einem Drittel wurde der Stuhltest angeboten, sie sollten innerhalb eines Jahres drei Stuhlproben abgeben. Einem weiteren Drittel wurde die Koloskopie empfohlen, das übrige Drittel hatte die Wahl zwischen beiden.

Wie zu erwarten, war die Akzeptanz der Koloskopie am geringsten: Nur 38\% nahmen das Angebot wahr, dagegen 67\% das eines Stuhltests. Interessant war nun das Ergebnis in der Gruppe mit der Wahlmöglichkeit: $69 \%$ entschieden sich für irgendeine Form des Screenings, und überraschenderweise war hier der Anteil derjenigen, die eine Koloskopie wählten, mit $31 \%$ nicht viel geringer als in der Gruppe derer, denen man nur die Koloskopie angeboten hatte.

Fazit: Wer bereit ist, eine Koloskopie machen zu lassen, tut dies offenbar auch, wenn man ihm einen Stuhltest als Alternative anbietet. Zusätzlich erreicht man über die Wahlmöglichkeit auch solche

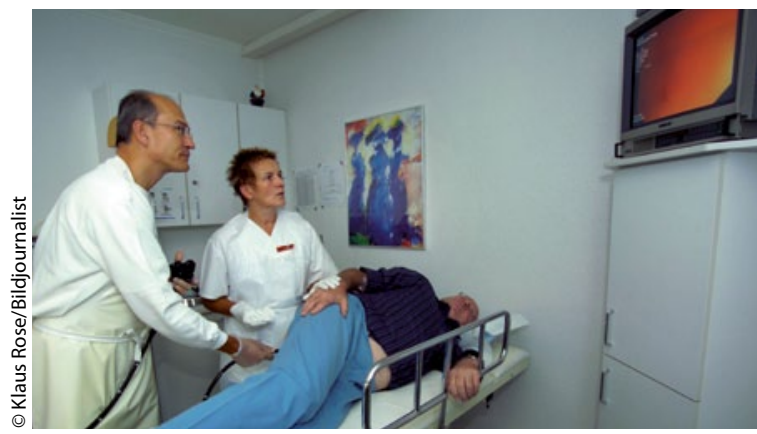

Viele Patienten scheuen die als unangenehm empfundene Koloskopie.

Leute, die eine Koloskopie ablehnen. Es ist es also günstiger, beide Optionen anzubieten, stellt auch der kalifornische Gastroenterologe Theodore Levin in einem Kommentar fest. Thomas Müller

Inadomi JM et al. Adherence to colorectal cancer screening: a randomized clinical trial of competing strategies. Arch Intern Med. 2012;172(7):575-82. - Kommentar: Levin TR et al. The importance of choosing colorectal cancer screening tests. Ibidem 582-3. 